\title{
Malaria Contracted in Pennsylvania
}

\author{
JOHN D. SHAW, M.P.H., and WILLIAM D. SCHRACK, Jr., M.D., Dr.P.H.
}

\begin{abstract}
LTHOUGH many health authorities beA lieve that malaria has been eradicated as an endemic disease in the United States, we have evidence indicating primary cases of malaria contracted by persons indigenous to the Commonwealth of Pennsylvania.

Malaria is never a static entity, least of all in the North Temperate Zone, either in its local manifestation or in its geographic distribution (1). To establish and maintain the disease, the malaria vector must be continuously present, and there must be infected persons and susceptible nonimmune persons accessible to attack by the vector.
\end{abstract}

Our purpose is to discuss briefly, for the public health worker, the conditions necessary for a malaria outbreak and to determine as closely as possible whether these factors are periodically present in the United States. Pennsylvania data will be used to illustrate concepts that are applicable to other areas of the United States. (The taxonomic terminology used in the identification of the life cycle of the Plasmodium parasite will be omitted whenever possible for the sake of clarity and simplicity.)

\section{Malaria Vector}

Anopheles quadrimaculatus (Say) is the most important vector of malaria in eastern North America (2). This mosquito, found throughout Pennsylvania, is an important potential vector in the Commonwealth (3). The establishment of the presence of the vector, however, is not significant in itself. The vector must take a blood meal containing plasmodia, must survive long enough for the parasite to develop

Mr. Shaw is a supervising sanitarian, division of sanitation, and Dr. Schrack is director, division of communicable diseases, Pennsylvania Department of Health. in its body, and then must bite a nonimmune person.

Horsfall (4) states that in areas inhabited by man A. quadrimaculatus may feed naturally on man, cows, horses, pigs, sheep, dogs, cats, and fowl. Examination of blood taken from mosquitoes of this species collected in human dwellings indicates that the majority of blood meals are on human hosts. Thus feeding seems to depend on the availability of the host. The females may seek a blood meal every third day, and their importance as malaria carriers to man depends on how frequently they select man under any given set of circumstances.

In nature most vector deaths are undoubtedly due to causes other than degeneration and lack of viability. They usually result from hazards of climate and natural enemies. Anophelines survive from fall to spring in caves and trees as evidenced by recent overwintering studies conducted in Pennsylvania ("1965 Distribution and Bionomics of Mosquitoes of Pennsylvania," unpublished paper by W. Wills, entomologist, division of sanitation, Pennsylvania Department of Health).

The time required for development of the parasite in the mosquito (extrinsic incubation period) depends upon environmental temperatures. A study by Thomas (5) of Plasmodium vivax infections in A. quadrimaculatus (fig. 1), shows that $82^{\circ} \mathrm{F}$. is the optimum temperature because it is the lowest temperature at which the development is completed in the shortest time. Other studies indicate that the optimum temperatures for the development of malaria parasites in the mosquito are approximately $(4,6)$ :

Plasmodium malariae- $72^{\circ} \mathrm{F}$.

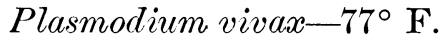

Plasmodium falciparum-86 ${ }^{\circ} \mathrm{F}$.

The average daily temperature per month for the 5-year period $1959-63$ in the 10 climatologic 
divisions of the State indicates that the highest temperatures are reached in July and August (7). A Pennsylvania Department of Health statewide mosquito survey, 1961-64, revealed that the adult population of $A$. quadrimaculatus is greatest during July and August. Figure 2 shows that the monthly temperatures for this period in many of the State's climatologic divisions will permit $P$. malariae to develop $\left(72^{\circ} \mathrm{F}\right.$. for $25-30$ days) and are sufficient for the development of $P$. vivax $\left(72.5^{\circ} \mathrm{F}\right.$. for 14 days).

The survival of the vector and parasite under these climatic and temporal conditions may be illustrated by this example. If 12 female $A$. quadrimaculatus mosquitoes feed on a person known to have $P$. vivax parasites in his or her blood, 6 mosquitoes, or 50 percent, may become infected with the parasite $(8)$. If the probable survival rate is 95 percent per day, at the end of a 14-day period approximately three of these six mosquitoes would be living and carrying the parasite at a stage in its development when it could cause malaria were the mosquitoes to take a human blood meal. If the probability of these six infected mosquitoes' surviving was 85 percent, the last mosquito would have died by the 12th day. Under Pennsylvania's climatic conditions, this is 2 days short of the time necessary for the parasite to develop in the mosquito. Thus we see that the percent survival per day is very important, particularly in geographic areas having temperatures low enough to require fairly long extrinsic incubation periods.

\section{Reservoir}

Although the malaria parasites may survive in the salivary glands of the mosquito for a long period, the insect seems to act mainly as a trans-

\section{Figure 1. Time-temperature relationship for development of Plasmodium vivax in Anopheles quadrimaculatus}

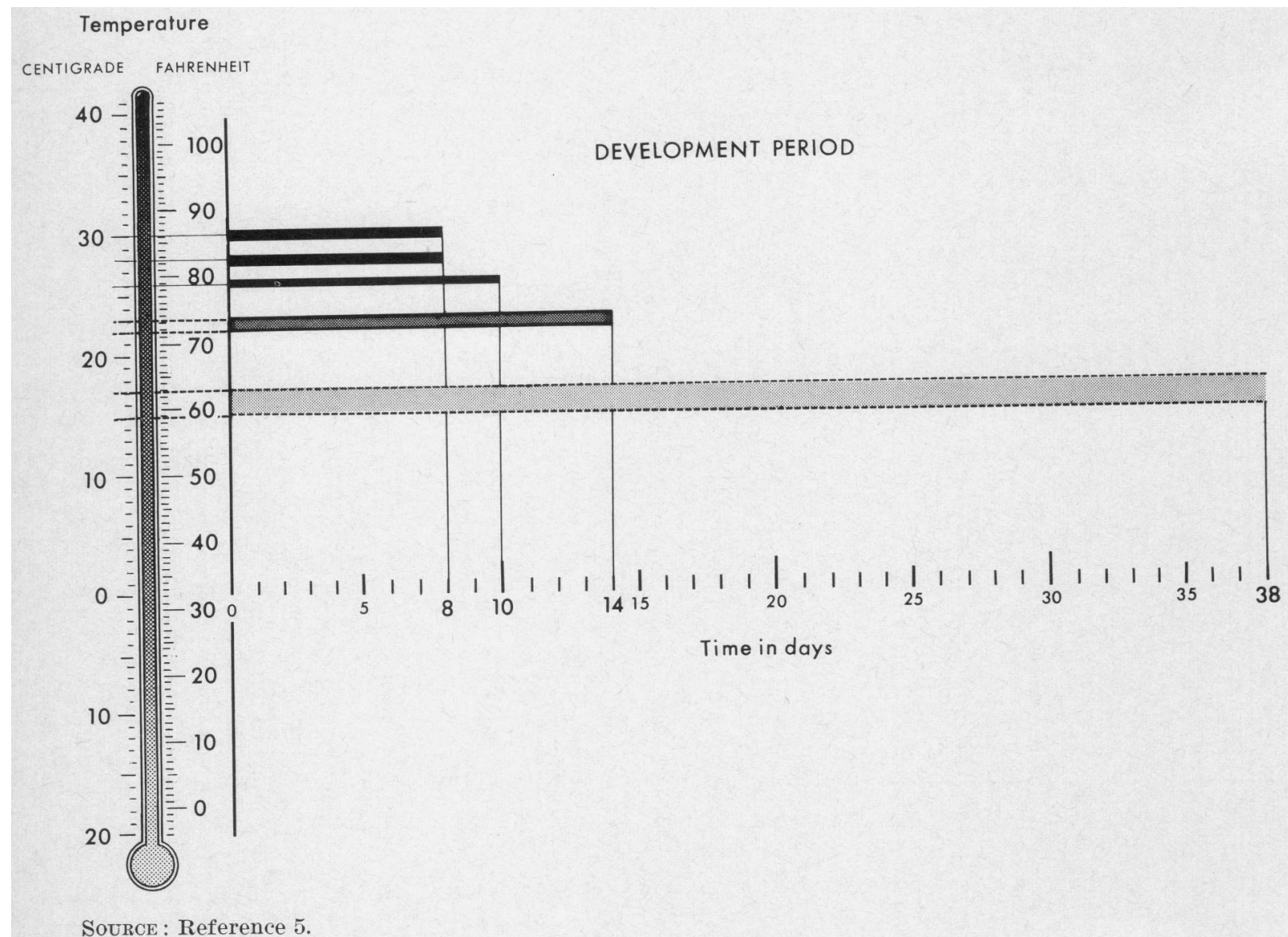


Figure 2. Climatic divisions of Pennsylvania with average monthly temperatures, 1959-63

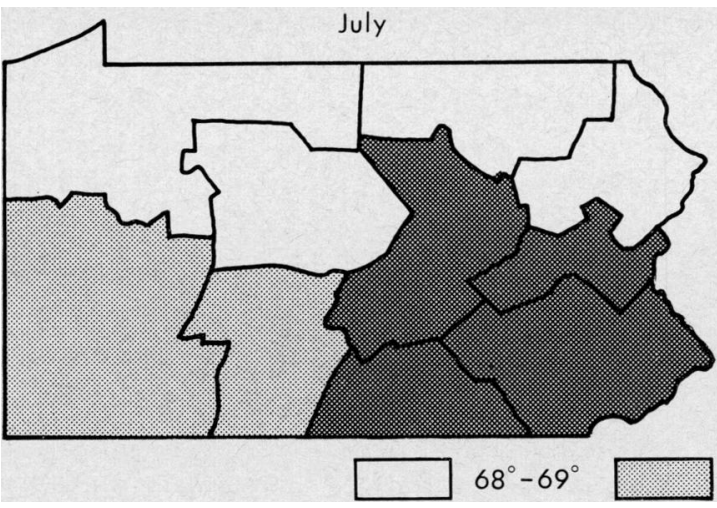

mitting agent and not as reservoir. Man is the reservoir of human malaria in North America. Since 1935 there have been 2,232 reported cases of malaria in Pennsylvania, the vast majority in military personnel stationed in the State during the years 1944 through 1947. The following data (based on 1935-64 records of the division of communicable disease control, Pennsylvania Department of Health) show the number of cases reported in Pennsylvania in this period:

\begin{tabular}{|c|c|}
\hline Year & Reported cases \\
\hline $1935-43 \ldots$ & 119 \\
\hline $1944 \ldots$ & 345 (2 civilians) \\
\hline 1945 & 663 (2 civilians) \\
\hline 1946 & $\begin{array}{l}876 \text { (6 civilians)-reported in } 33 \\
\text { counties. }\end{array}$ \\
\hline $1947 \ldots$ & 137 (2 civilians) \\
\hline $1948 \ldots$ & 23 \\
\hline 1949 & 27 \\
\hline $1950 \ldots$ & 1 \\
\hline $1951 \ldots \ldots$ & 2 \\
\hline $1952-62 \ldots$ & 23 \\
\hline $1963 \ldots \ldots$ & $\begin{array}{l}5 \text { (1 indigenous to Allegheny } \\
\text { County). }\end{array}$ \\
\hline $1964 \ldots$ & 11 \\
\hline
\end{tabular}

In 1945 one case of malaria was acquired in Lehigh County and one in Northampton County. In 1946 and again in 1948 one male civilian patient was treated for malaria in Montgomery County. It was believed that he had contracted the disease while in Huntingdon, Pa. H. D. Propst, M.D., Honesdale, Pa. (in a letter to the Pennsylvania Department of Health, June 22, 1953), referred to 6 cases of malaria previously reported in patients who had never been out of northern Pennsylvania and New York State.

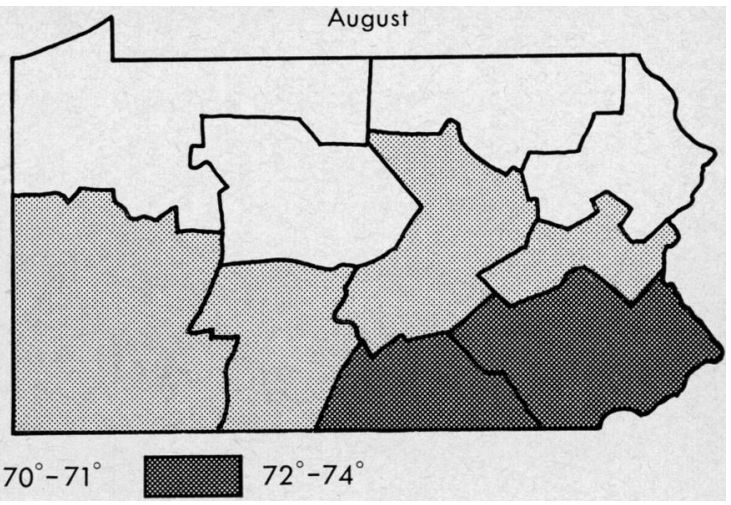

In July 1963, a 56-year-old woman resident of Pittsburgh first experienced chills which recurred every fourth day. In late October, physical examination revealed her spleen to be enlarged. Upon a routine complete blood count performed in November, a laboratory technician noted malaria parasites. The slide was read as positive for $P$. malariae at the hospital and confirmed at the Communicable Disease Center, Public Health Service, Atlanta, Ga. (9). The patient was born in Czechoslovakia and at age $1 \frac{1}{2}$ had immigrated to the United States. She had never been known to have had malaria earlier. Her rare travels had been confined to the northeastern United States. She denied having been outside Allegheny County in the past decade and denied a history of drug addiction. (The needle used to inject drugs has been incriminated in the spread of malaria from one addict to another.) The woman stated she had never received any blood transfusions. A check of 33 regional hospitals and blood banks revealed no record of a blood transfusion to this woman.

The number of persons currently living in Pennsylvania who have at one time had malaria and who might have a reappearance of, or a marked increase in the number of circulating parasites (relapse) is unknown. Some of the 2,232 persons with reported cases are in this category. Furthermore, some cases of malaria may have escaped the attention of the physician or not have been recognized because the disease seemingly had disappeared in this State for a number of years. It is equally difficult to estimate the number of cases not reported because the primary attacks occurred in another State 
or outside the United States and no apparent relapses had occurred since the person entered the Commonwealth. An African student in an American college or university who has had malaria may be unaware that he has had this disease and may never display the outward signs associated with a relapse. Periodically, however, the parasite will be found in the student's circulatory system in a high enough concentration to permit transmission if the proper vector and host are present.

The importance of one person as a source of infection was well established by Brunetti and associates (10), who reported that 35 cases of $P$. vivax malaria occurred in 1952 among a group of Camp Fire girls attending summer camp at Lake Vera, Calif. The probable source of these infections was traced to a recently returned Korean veteran. This outbreak also demonstrates the vulnerability of a nonimmune population when favorable conditions exist and malaria is introduced from abroad.

Russell and associates (11) state that relapses are uncommon in Plasmodium ovale infections and fairly common in cases caused by $P$. falciparum, but as a rule they do not occur more than 1 year after infection. The authors further state that $P$. malariae is known for its relapsing character and that undoubtedly $P$. malariae relapses have occurred 30 years or more after infection. Often the only clue to a continuing $P$. malariae infection is a case of malaria occurring promptly after a blood transfusion from a previously unsuspected carrier of the parasite, a phenomenon first reported in Pennsylvania in 1946 (12). The most notorious of the four species of Plasmodium for relasping tendencies is $P$. vivax, but in most cases no relapses occur after $21 / 2$ years. Russell and associates note, however, that relapses have been observed in the third year after a $P$. vivax infection and in occasional cases for 1 or 2 years longer. When the parasite's ability to cause relapses increases, the importance of relapses as active reservoirs also increases.

\section{Discussion}

The extremely low incidence of malaria in Pennsylvania aids in maintaining a high level of susceptibility in the population. Some of the military personnel returning to the States at the end of World War II and the Korean conflict were potential reservoirs of infection. Tourists returning to the United States from subtropical and tropical areas may carry the malaria infection. Not all patients with malaria are treated with drugs that will prevent relapse. And, like the unsuspecting African student and the rare person with a case of malaria that has escaped the attention of a physician, they are all potential reservoirs of infection. It must be emphasized, however, that this reservoir is extremely small and at times may be nonexistent, since it depends on the number, frequency, and duration of relapses.

The mosquito survey of the Pennsylvania Department of Health demonstrated clearly the statewide distribution of $A$. quadrimaculatus, the most important vector of malaria in eastern United States. This mosquito, found in all climatic divisions of the State, was shown to have peak adult populations during July and August, the months of the highest average daily temperature. Laboratory temperatures are sufficient to permit the cyclic development in the mosquito of $P$. malariae within 25-30 days and of $P$. vivax within 14 days. Laboratory temperatures, however, may not reflect the fluctuating temperature prevalent in the natural environment of the adult mosquito. A. quadrimaculatus is zoophilic, but whether it feeds on man depends upon man's availability.

In Pennsylvania, a susceptible population is periodically exposed to an indigenous or migrating reservoir of infection in a geographic area that affords both the vector and the climatic conditions necessary for transmission of malarial infection. Then why have only a few cases of the disease been reported? The possibility of unrecognized cases probably is of minor significance in explaining the extremely low incidence of reported infection. One of the most limiting factors is the small population of A. quadrimaculatus in the State. The fairly long extrinsic incubation periods of the malaria parasite and the zoophilic feeding habits of the mosquito necessitate certain conditions to insure transmission of the malaria infection from man to man : $(a)$ active feeding by the mosquitoes on a reservoir, (b) a high mosquito survival rate, and $(c)$ active feeding by large numbers of 
mosquitoes on a susceptible population. In geographic areas of the United States experiencing slightly higher average daily temperatures than Pennsylvania the extrinsic incubation period will be completed in fewer days. In such areas, fewer mosquitoes with lower survival rates can perpetuate the malaria infection.

Proper design, construction, and maintenance of water impoundments is to a large extent responsible for the small A. quadrimaculatus population in Pennsylvania. Tre have further protected ourselves by screening the doors and windows of our dwellings. These measures have helped isolate the reservoir from the vector and subsequently, the vector from the susceptible population. Powell and Brewer (13) state that the increasing resistance to residual insecticides by the vector and increasing evidence of resistance to antimalarial drugs by the parasite are well established. The potential of active immunization against malaria in man has not been completely explored. Man's ability to control malaria by controlling his environment, however, is well established.

We can no longer consider the flight range of the Anopheles mosquito as a barrier protecting man from its bite and the subsequent possibility of disease. For man in his desire for more space and more time for outdoor sports has moved into an environment somewhat foreign to himself and potentially favorable to the vector of malaria.

The Pennsylvania Department of Health's first publication on vector control, "The Malarial Mosquito and the Rain Barrel Mosquito," published in 1907 (14), stated: "No one can get malaria unless bitten by the malarial mosquito, hence the necessity for its extermination." Today, some 58 years later, this concept still applies, not only to Pennsylvania but to all of the United States.

\section{Summary}

Indigenous and immigrating reservoirs of human malaria parasites exist periodically in Pennsylvania in the midst of a susceptible population. Moreover, adult mosquitoes of the species Anopheles quadrimaculatus are found in all 10 climatic divisions of the State; peak populations of the species have been observed in July and August. In July and August the average daily temperature in Pennsylvania will permit completion of the extrinsic incubation period of Plasmodium malariae within 25-30 days and of Plasmodium vivax within 14 days. The presence of the mosquito, its proximity to man, and its feeding habits make it possible under these climatic conditions for the mosquito to transmit malaria from a reservoir to a susceptible population. The few cases of reported malaria in Pennsylvania provide further evidence that all conditions necessary for transmission of this disease are periodically present in the State and that transmission does occur.

\section{REFERENCES}

(1) Federal Security Agency, U.S. Public Health Service, and Tennessee Valley Authority: Malaria control on impounded water. U.S. Government Printing Office, Washington, D.C., 1947, pp. 2-7.

(2) Foote, R. H., and Cook, D. R.: Mosquitoes of medical importance. U.S. Department of Agriculture Handbook No. 152. U.S. Government Printing Office, Washington, D.C., July 1959, p. 133.

(3) Shaw, J. D., and Wills, W.: Abundance and distribution of potential mosquito vectors in Pennsylvania. Paper presented at 12 th annual health conference, 1963. Pennsylvania Department of Health, Harrisburg, Pa., 1963.

(4) Horsfall, W. R.: Mosquitoes-their bionomics and relation to disease. The Ronald Press Company, New York, 1955, pp. 51-56 and 149.

(5) Stratman-Thomas, W. K.: The influence of temperature on Plasmodium vivax. Amer J Trop Med 20: 703-715, September 1940.

(6) Bates, M.: The natural history of mosquitoes. The Macmillan Company, New York, 1949, pp. 231-239.

(y) U.S. Department of Commerce Weather Bureau : Climatological data, Pennsylvania, June, July, August, and September 1959-63.

(8) Macdonald, G.: The epidemiology and control of malaria. London Oxford University Press, Inc., London, 1957, pp. 6-10.

(9) Communicable Disease Center, U.S. Public Health Service: Morbidity and Mortality Weekly Report Vol. 13, No. 3, Jan. 24, 1964, p. 22.

(10) Brunetti, R., Fritz, R. F., and Hollister, A. C., Jr. : An outbreak of malaria in California, 1952-53. Amer J Trop Med 3: 779-788, September 1954.

(11) Russell, P. F., West, L. S., Manwell, R. D., and Macdonald, G.; Practical malariology. Ed. 2. 
Oxford University Press, Inc., New York, 1963, pp. $425-432$.

(12) Schrack, W. D., Jr.: Malaria infection acquired through blood transfusion. Public Health Rep 61 : 1630 , Nov. 8, 1946.

(13) Powell, R. D., and Brewer, G. J. : Active immuni- zation against malaria. Supp. to Amer J Trop Med 13 : 228-232, January 1964.

(14) Pennsylvania Department of Health: The malaria mosquito and the rain barrel mosquito. Harrisburg Publishing Co., Harrisburg, Pa., 1907, p. 3.

\section{Public Health Service Staff Appointments}

Dr. Eugene H. Guthrie, recently appointed Assistant Surgeon General for Operations, has moved to third in line of command within the Public Health Service. He succeeds Assistant Surgeon General James M. Hundley who retired January 31, 1966.

Born in Washington, D.C., Dr. Guthrie has spent his entire professional career in the Public Health Service. He received his medical degree from the George Washington University School of Medicine in 1951 and his master's degree in public health from the University of Michigan in 1955. He was commissioned in the Service in 1951 when he began his internship at the Public Health Service Hospital in Baltimore. Subsequently, he was public health resident in the Montgomery County (Md.) Health Department and in the California State Department of Public Health.

Dr. Guthrie came to Washington in 1956 as chief of School Health and Rural Health Activities. In 1959, he was named chief program officer for the Bureau of State Services, and, 2 years later, chief of the Neurological and Sensory Disease Service Branch of the Division of Chronic Diseases. Several months later he became chief of the Division. He received the Public Health Service Meritorious Medal in 1964. for his work on the Surgeon General's Advisory Committee on Smoking and Health.

Dr. Guthrie is a member of the American Medical Association, American Public Health Association, American School Health Association, and the Medical Committee of the President's Commission on Employment of the Handicapped.

Dr. Francis A. Arnold, Jr., formerly director of the National Institute of Dental Research, succeeded Assistant Surgeon General Ralph S. Lloyd, who retired February 1, 1966, as Chief Dental Officer.

Dr. Arnold, born in Orrville, Ohio, received his dental degree from Western Reserve University in 1934. He served his internship at the U.S. Marine
Hospital in Cleveland and was commissioned in the Public Health Service in 1936. He has been in dental research at the National Institutes of Health since 1937 and has served as associate director and director. A pioneer in the study of fluorides and epidemiologic studies of fluoridation, Dr. Arnold received an honorary doctor of science degree from Western Reserve University and the Callahan Award, the Geis Award, and the Dean Award. He is a Fellow of the American College of Dentists and the American Public Health Association and a member of the International Association for Dental Research, American Dental Association, American Epidemiological Society, American Association for the Advancement of Science, Washington Academy of Medicine, and Washington Academy of Science.

Dr. David J. Sencer, whose entire professional career has been in the area of infectious disease control, has been named chief of the Communicable Disease Center, Atlanta, Ga. He succeeds Assistant Surgeon General James L. Goddard who was appointed Commissioner of the Food and Drug Administration.

A native of Grand Rapids, Mich., Dr. Sencer was educated at Wesleyan University, Middleton, Conn., and the University of Mississippi and received his medical degree from the University of Michigan in 1951. He served his internship and his residency in internal medicine at the University of Michigan Hospital, and received his master's degree in public health from Harvard University in 1958.

Dr. Sencer has been a member of the Public Health Service Commissioned Corps since 1955. He has also served as assistant chief and deputy chief of the Communicable Disease Center. He is a member of the American Medical Association, American Public Health Association, American Thoracic Society, and American Society of Tropical Medicine. 